\title{
Fano effect in a double T-shaped interferometer
}

\author{
V. Moldoveanu ${ }^{1, a}$, I.V. Dinu ${ }^{1}$, and B. Tanatar ${ }^{2}$ \\ 1 National Institute of Materials Physics, P.O. Box MG-7, 077125 Bucharest-Magurele, Romania \\ 2 Department of Physics, Bilkent University, Bilkent, 06800 Ankara, Turkey
}

Received 25 August 2008 / Received in final form 10 November 2008

Published online 20 January 2009 - (c) EDP Sciences, Società Italiana di Fisica, Springer-Verlag 2009

\begin{abstract}
We study the coherent transport in a one-dimensional lead with two side-coupled quantum dots using the Keldysh's Green function formalism. The effect of the interdot Coulomb interaction is taken into account by computing the first and second order contributions to the self-energy. We show that the Fano interference due to the resonance of one dot is strongly affected by the fixed parameters that characterize the second dot. If the second dot is tuned close to resonance an additional peak develops between the peak and dip of the Fano line shape of the current. In contrast, when the second dot is off-resonance and its occupation number is close to unity the interdot Coulomb interaction merely shifts the Fano line and no other maxima appear. The system we consider is more general than the single-dot interferometer studied experimentally by Kobayashi et al. [Phys. Rev. B 70, 035319 (2004)] and may be used for controlling quantum interference and studying decoherence effects in mesoscopic transport.
\end{abstract}

PACS. 73.23.Hk Coulomb blockade; single-electron tunneling - 85.35.Ds Quantum interference devices 85.35.Be Quantum well devices - 73.21.La Quantum dots

\section{Introduction}

The level structure of quantum dot systems is very sensitive to the potentials applied on the metallic gates that define them. In particular, one can match a discrete level of the quantum dot to the continuous spectrum of the incident electrons from leads. This tunability is nowadays widely used to study resonant transport and quantum interference effects in various structures (for recent reviews see Refs. [1,2]). A typical example is the experiment of Kobayashi et al. [3] in which the measured conductance of a quantum wire with a side-coupled quantum dot shows asymmetric Fano line shapes [4] as a function of the gate potential $V_{g}$ applied on the latter. This effect is due to the interference between two types of electronic trajectories within the system: the forward scattered electronic waves and the more complicated paths involving scattering at the dot, i.e., resonant tunnelling processes. A similar effect was previously observed in single-dot Aharonov-Bohm rings [5].

The recent observation of the Fano-Kondo effect [6] in mesoscopic interferometers with a side-coupled quantum dot stimulated many theoretical investigations on strongly correlated transport. Various non-perturbative methods were used to study the formation of the Kondo correlated states in systems composed of a quantum dot which on one hand is coupled to two leads and on the other hand has a second side-coupled dot.

\footnotetext{
a e-mail: valim@infim.ro
}

Kim and Hershfield [7] considered the electronic transport through such a system by including the on-site Coulomb interaction in the side-coupled dot, while neglecting both the interdot interaction and the Coulomb repulsion on the central dot. The interaction self-energy was computed within the non-crossing approximation (NCA) and the spectral functions of the two dots were analyzed. The Kondo scattering was shown to reduce the conductance of the system at low bias. Franco et al. [8] used the $\mathrm{X}$-boson method for the single-impurity model in order to compute the conductance of side-coupled dot systems for weak and strong lateral coupling. Later on Cornaglia and Grempel [9] studied the same system using both numerical renormalization group techniques (NRG) and slaveboson mean-field theory (SBMFT). The temperature dependence of the conductance was discussed for the case in which the dots are half-filled or when the total charge of the dot is odd or even. We stress that in the approach considered there the Coulomb interaction exceeds all the tunneling couplings appearing in the problem and therefore the main physical processes are due to spin fluctuations, the occupation on each dot being close to unity.

Another interacting regime was investigated by $\mathrm{Wu}$ et al. [10] still within SBMFT. In their model the central dot (i.e., the one which is connected to the leads) operates in the Kondo regime, the side-coupled dot being instead noninteracting. The formula for the density of states was decomposed into a broad Breit-Wigner resonance term and a Fano line shape contribution. A comparison between interacting and noninteracting Fano line shapes of the current in a $T$-network was presented by 


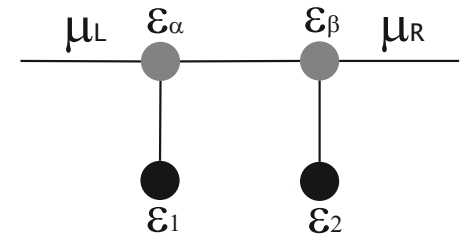

Fig. 1. A schematic representation of the double $T$-shaped interferometer. The 4-site structure is coupled to two semiinfinite leads with chemical potentials $\mu_{L}$ and $\mu_{R}$. Electronelectron interactions are allowed only between the side-coupled dots (dark sites). The other two (grey sites) are noninteracting and provide the 'background' component in the transport.

Aharony et al. [11] In the case of an infinite interaction it turns out that a strong destructive interference appears at $T=0$. In a recent work [12] the correlation functions for a strongly correlated double quantum dot were also computed within the renormalization group approach.

In this work we consider the Fano effect in a more complex system, namely a double $T$-shaped interferometer composed of a one-dimensional lead to which two sidecoupled dots are attached (see Fig. 1 for a schematic description). We also focus on the strong coupling regime with respect to the leads and take into account the interdot Coulomb interaction. Such a system can also be easily realized in experiments and should provide important insights on the control of quantum interference and on the decoherence induced by the mutual Coulomb interaction between the dots. In the case of the double $T$-shaped interferometer the possibility of individually tuning the quantum dot levels is expected to bring different and more complex transport regimes, as each dot can be set to interfere with the forward scattered electrons in the lead. Studying this interplay is the main goal of this work.

We wish to mention also that the effect of electronelectron interaction on the Fano interference in a double quantum dot coupled to two leads in a parallel configuration was investigated recently in a series of papers $[13,16]$ for various regimes. Within the equation of motion method Sztenkiel et al. [13] used both the Hartree-Fock approximation (valid at temperatures higher than the Kondo temperature) and the $\mathrm{Ng}$ ansatz (valid at low temperatures) to compute the spectral densities and the conductance of this system. They have focused on the Kondo spinless anomaly and emphasized the role of the interdot coupling in the Fano interference. The Kondo regime for single-dot Aharonov-Bohm interferometer was considered within a perturbative approach by Son et al. [14] The role of infinite intradot interaction and long-range Coulomb interaction in $A B$ interferometers with two embedded dots was considered within a slave-boson approach by Ma et al. [15]

We believe that our perturbative approach on the interaction effects in a double $T$-shaped interferometer should complement the existing results, especially in the context of decoherence induced by inelastic scattering processes which cannot be captured in a mean-field approach.

The paper is organized as follows: Section 2 sets the notation and gives the relevant transport equations within the Keldysh formalism. Section 3 presents the numerical results and their discussion. We conclude in Section 4.

\section{Formalism}

At the theoretical level, the quantum transport in interacting nanostructures is suitably described within the Keldysh's Green function formalism $[17,18]$ which provides, along a well established scheme, the steady-state current through a mesoscopic system $(S)$ which is adiabatically coupled to noninteracting biased leads $(L)$ in the remote past. Since in the long-time limit the initial correlations are negligible, at the formal level the electronelectron interaction and the coupling to the leads are both established in the remote past [18]. Then the Hamiltonian quite generally reads:

$$
H(t)=H_{S}+H_{L}+\chi(t)\left(H_{T}+H_{I}\right),
$$

where $\chi(t)$ is a smooth switching function which is chosen such that $\chi(-\infty)=0$ and $\chi(t)=1$ for $t>0$.

The system we shall consider here is sketched in Figure 1 and is described by the following tight-binding Hamiltonian (h.c. denotes Hermitian conjugate and n.n indicates nearest neighbor summation):

$$
\begin{aligned}
H_{S}= & \sum_{i=1,2} \varepsilon_{i} a_{i}^{\dagger} a_{i}+\sum_{\nu=\alpha, \beta} \varepsilon_{\nu} a_{\nu}^{\dagger} a_{\nu} \\
& +\left(t_{L} a_{\alpha}^{\dagger} a_{\beta}+\sum_{i, \nu \in \mathrm{n} . \mathrm{n}} t_{i \nu} a_{i}^{\dagger} a_{\nu}+\text { h.c. }\right) \\
H_{T}= & t_{L}\left(c_{0_{L}}^{\dagger} a_{\alpha}+c_{0_{R}}^{\dagger} a_{\beta}+h . c\right), \quad H_{I}=U a_{1}^{\dagger} a_{1} a_{2}^{\dagger} a_{2} \\
H_{L}= & \sum_{l=L, R} \sum_{m, n \in \mathrm{n} . \mathrm{n}} t_{L}\left(c_{m_{l}}^{\dagger} c_{n_{l}}+h . c\right) \quad m_{l}, n_{l}=0, \ldots \infty .(4)
\end{aligned}
$$

$H_{S}$ describes a non-interacting 4 -site central region. The lower sites describe single-level quantum dots and $\varepsilon_{i}(i=$ $1,2)$ is the on-site energy. Each quantum dot is sidecoupled to another site $\nu(\nu=\alpha, \beta)$ which in turn is connected to a semi-infinite lead via the tunneling term $H_{T}$. The operators $a^{\dagger}, a$ create and annihilate particles in the central system, respectively. $c_{0_{L}}^{\dagger}, c_{0_{R}}^{\dagger}$ are creation operators in the endpoint sites $0_{L}$ and $0_{R}$ of the left $(\mathrm{L})$ and right $(\mathrm{R})$ leads that connect to the system. $t_{i \nu}$ are hopping coefficients, $U$ is the interaction strength between the quantum dots and $t_{L}$ is the hopping energy on leads and between the sites $\alpha$ and $\beta$. The transport is generated by a finite bias applied on the leads which are described by the Hamiltonian $H_{L}$. As usual this bias is introduced as the difference between the chemical potentials of the two leads $e V=\mu_{L}-\mu_{R}$. For simplicity we take the on-site energy of the leads to be zero.

In actual experiments the two dots can be quite close to one another such that the Coulomb interaction is expected to modify transport properties. In this work we shall consider only the effect of the interdot electron-electron 
interaction while neglecting the interaction between the electrons in the dots and the ones in the wire (we discuss this aspect further in Sect. 2). We mention that in this setup the dots are coupled to the lead in different locations, but we could also consider an alternative geometry, in which the dots are placed symmetrically with respect to the lead and share the same contact site.

A standard application of the Langreth rules leads to the following formula for the steady-state current in the left lead (see [19] for details):

$$
\begin{aligned}
J_{L}= & \frac{e t_{L}^{2}}{\hbar} \int_{-2 t_{L}}^{2 t_{L}} d E\left(2 \pi \rho^{2}\left|G_{\alpha \beta}^{R}\right|^{2}\left(f_{L}-f_{R}\right)\right. \\
& -\rho\left(G^{R} \operatorname{Im}\left(2 \Sigma_{I}^{R} f_{L}+\Sigma_{I}^{<}\right) G^{A}\right)_{\alpha \alpha},
\end{aligned}
$$

where $G^{R}$ is the $4 \times 4$ matrix of the retarded Green function of the coupled and interacting system and $\rho(E)=$ $\sqrt{4 t_{L}^{2}-E^{2}} /\left(2 \pi t_{L}^{2}\right)$ is the density of states at the endpoint of the leads. For simplicity we omit showing the energy dependence in equation (5). The second term in the current formula is the diagonal element of a matrix product containing the imaginary parts of the retarded and lesser interaction self-energy $\Sigma_{I}$. One can easily recognize a Landauer form in the first term in equation (5), in spite of the fact that the Green function there embodies both the interaction and leads' self-energies. The second term is a nontrivial correction whose effect in dephasing was discussed in our recent works $[19,20] . f_{L}$ and $f_{R}$ are the Fermi functions in the left and right leads, respectively.

We further denote by $G_{0}^{R,<}$ the retarded and lesser noninteracting Green functions of the coupled system. These quantities can be easily computed by taking advantage of the fact that the retarded and lesser leads' selfenergies are known (note that only the diagonal elements of $\Sigma_{L}$ are nonvanishing and that $\left.E \in\left[-2 t_{L}, 2 t_{L}\right]\right)$ :

$$
\begin{aligned}
& \Sigma_{L, \alpha \alpha}^{R}=\Sigma_{L, \beta \beta}^{R}=\frac{1}{2 t_{L}^{2}}\left(E-i \sqrt{4 t_{L}^{2}-E^{2}}\right), \\
& \Sigma_{L, \alpha \alpha}^{<}=2 \pi i t_{L}^{2} \rho(E) f_{L}(E), \Sigma_{L, \beta \beta}^{<}=2 \pi i t_{L}^{2} \rho(E) f_{R}(E) .
\end{aligned}
$$

At the second step the interaction self-energy is computed perturbatively up to the second order in the interaction strength $U$, namely $\Sigma_{I}=\Sigma_{I}^{1}+\Sigma_{I}^{2}$. A standard analysis of the Feynman diagrams for the contour-ordered Green functions gives (the bar denotes complex conjugation):

$$
\begin{aligned}
\Sigma_{I, 12}^{R, 1}= & \frac{i U}{2 \pi} \int d E^{\prime} G_{0,12}^{<}\left(E-E^{\prime}\right)=\bar{\Sigma}_{I, 12}^{R, 1} \\
\Sigma_{I, 11}^{R, 1}= & -\frac{i U}{2 \pi} \int d E^{\prime} G_{0,22}^{<}\left(E^{\prime}\right), \\
\Sigma_{I, 22}^{R, 1}= & -\frac{i U}{2 \pi} \int d E^{\prime} G_{0,11}^{<}\left(E^{\prime}\right) \\
\Sigma_{I, i j}^{<, 1}= & \Sigma_{I, i \nu}^{<, 1}=\Sigma_{I, \mu \nu}^{<, 1}=0, \quad i, j=1,2 \quad \mu, \nu=\alpha, \beta, \\
\Sigma_{I}^{<, 2}= & \frac{U^{2}}{2 \pi^{2}} \int d E_{1} d E_{2} \\
& \times G_{0}^{<}\left(E-E_{1}\right) G_{0}^{<}\left(E_{2}\right) G_{0}^{>}\left(E_{2}-E_{1}\right) .
\end{aligned}
$$

We remark that the diagonal elements of the first order interacting self-energy are real and that $\Sigma_{I, 12}^{R}$ has also an imaginary part but does not depend on energy (the dependence on $E$ can be eliminated by a change of variable). The lesser Green function in the above equations is to be computed from the Keldysh equation $G_{0}^{<}=G_{0}^{R} \Sigma_{L}^{<} G_{0}^{A}$. The second order retarded self-energy is related to the lesser and greater self-energies by the identity (see [21] ):

$$
\Sigma_{I}^{R, 2}(E)=\lim _{\epsilon \rightarrow 0+} \frac{i}{2 \pi} \int d E^{\prime} \frac{\Sigma_{I}^{>, 2}\left(E^{\prime}\right)-\Sigma_{I}^{<, 2}(E)}{E-E^{\prime}+i \epsilon} .
$$

Using the Dyson equation for the retarded Green function of the central region $G^{R}=G_{0}^{R}+G_{0}^{R} \Sigma_{I}^{R} G^{R}$ one can write $G^{R}=\left(H_{\text {eff }}-z\right)^{-1}$ where we introduce the following effective single-particle Hamiltonian:

$$
H_{\mathrm{eff}}=\left(\begin{array}{cccc}
\varepsilon_{1}-\Sigma_{I, 11}^{R} & -\Sigma_{I, 12}^{R} & t_{1 \alpha} & 0 \\
-\Sigma_{I, 21}^{R} & \varepsilon_{2}-\Sigma_{I, 22}^{R} & 0 & t_{2 \beta} \\
t_{\alpha 1} & 0 & \varepsilon_{\alpha}-t_{L}^{2} e^{-i k} & t_{L} \\
0 & t_{\beta 2} & t_{L} & \varepsilon_{\beta}-t_{L}^{2} e^{-i k}
\end{array}\right) .
$$

The exponential terms in the lower right $2 \times 2$ block are due to the leads' self-energy and $k$ is the momentum of the incident electrons from leads, defined by the usual relation $E=2 t_{L} \cos k$. The upper left $2 \times 2$ block describes the interacting isolated dots. In order to discuss the various tunneling processes in the system we write down the relevant Green function $G_{\alpha \beta}^{R}$ using the blockwise inversion formula:

$$
\left(\begin{array}{ll}
A & B \\
C & D
\end{array}\right)^{-1}=\left(\begin{array}{cc}
M^{-1} & -M^{-1} B D^{-1} \\
-D^{-1} C M^{-1} & D^{-1}+D^{-1} C M^{-1} B D^{-1}
\end{array}\right)
$$

where $M=A-B D^{-1} C$ is the so-called Schur complement of the lower right block $D$. We shall apply this formula for $\left(H_{\text {eff }}-E\right)^{-1}$. To this end we need a few more notations. We first define

$$
G^{b}=\left(\begin{array}{cc}
\left(\tilde{\varepsilon}_{\alpha}-E\right)^{-1} & -t_{L} \Delta^{-1} \\
-t_{L} \Delta^{-1} & \left(\tilde{\varepsilon}_{\beta}-E\right)^{-1}
\end{array}\right)=:\left(\begin{array}{c}
G_{\alpha \alpha}^{b} G_{\alpha \beta}^{b} \\
G_{\beta \alpha}^{b} G_{\beta \beta}^{b}
\end{array}\right),
$$

with

$$
\begin{aligned}
\Delta & =\left(\varepsilon_{\alpha}-t_{L}^{2} e^{-i k}-E\right) \cdot\left(\varepsilon_{\beta}-t_{L}^{2} e^{-i k}-E\right)-t_{L}^{2}, \\
\tilde{\varepsilon}_{\alpha} & =\varepsilon_{\alpha}-t_{L}^{2} e^{-i k}-t_{L}^{2}\left(\varepsilon_{\beta}-t_{L}^{2} e^{-i k}-E\right)^{-1}, \\
\tilde{\varepsilon}_{\beta} & =\varepsilon_{\beta}-t_{L}^{2} e^{-i k}-t_{L}^{2}\left(\varepsilon_{\alpha}-t_{L}^{2} e^{-i k}-E\right)^{-1} .
\end{aligned}
$$

$G^{b}$ is the inverse of the upper left $2 \times 2$ block in the matrix $H_{\text {eff }}-E$ and describes the forward scattering of electrons (i.e., the so-called background signal, which does not depend either on $\varepsilon_{1}$ or on $\varepsilon_{2}$ ). The renormalized energies of the contact sites $\tilde{\varepsilon}_{\alpha, \beta}$ contain the leads self-energy and also the effect of the coupling between the two sites. Note that the coupling to the leads introduces an imaginary part 
in the denominators of $G^{b}$ so that there is no singularity there.

The Schur complement is defined as $G^{d}=\left(H^{d}-z\right)^{-1}$, and the Hamiltonian $H^{d}$ has the following form:

$$
H^{d}=\left(\begin{array}{cc}
\tilde{\varepsilon}_{1} & -\Sigma_{I, 12}^{R}-t_{1 \alpha} G_{\alpha \beta}^{b} t_{\beta 2} \\
-\Sigma_{I, 21}^{R}+t_{2 \beta} G_{\beta \alpha}^{b} t_{\alpha 1} & \tilde{\varepsilon}_{2}
\end{array}\right),
$$

in which we introduced the notations:

$$
\begin{aligned}
& \tilde{\varepsilon}_{1}=\varepsilon_{1}-\Sigma_{I, 11}^{R}-t_{1 \alpha} G_{\alpha \alpha}^{b} t_{\alpha 1} \\
& \tilde{\varepsilon}_{2}=\varepsilon_{2}-\Sigma_{I, 22}^{R}-t_{2 \beta} G_{\beta \beta}^{b} t_{\beta 2} .
\end{aligned}
$$

From the physical point of view, $G^{d}$ is an effective Green function of the two side-coupled dots which takes into account the effect of the lead-dot coupling and of the interaction. From the diagonal elements one infers that the levels of the disconnected dots become resonances when the dots are side-coupled to the leads. Then the Green function $G_{\alpha \beta}^{R}$ that enters the equation for the current is the sum of several terms, each of them describing a class of electronic trajectories:

$$
\begin{aligned}
G_{\alpha \beta}^{R}= & G_{\alpha \beta}^{b}+G_{\alpha \alpha}^{b} t_{\alpha 1} G_{11}^{d} t_{1 \alpha} G_{\alpha \beta}^{b}+G_{\alpha \beta}^{b} t_{\beta 2} G_{22}^{d} t_{2 \beta} G_{\beta \beta}^{b} \\
& +G_{\alpha \alpha}^{b} t_{\alpha 1} G_{12}^{d} t_{2 \beta} G_{\beta \beta}^{b}+G_{\alpha \beta}^{b} t_{\beta 2} G_{21}^{d} t_{1 \alpha} G_{\alpha \beta}^{b} .
\end{aligned}
$$

In order to have a more intuitive picture of the interference effects one should write first the Schur complement as $G^{d}=\left(H_{0}^{d}+T-z\right)^{-1}$ where $H_{0}^{d}$ is the diagonal part of $H_{d}$ and $T$ is given by the off-diagonal part. If we introduce the notation $G_{0}^{d}=\left(H_{0}^{d}-z\right)^{-1}$ a Dyson expansion for $G^{d}$ in terms of $T$ reads:

$$
G^{d}=G_{0}^{d}+G_{0}^{d} T G_{0}^{d}+\ldots
$$

Replacing this structure in equation (22) one recovers all electronic trajectories within the $T$-shaped system. For example, the first term in $G^{d}$ describes single tunneling processes in which electrons entering the system from the left lead tunnel through one dot only, before escaping into the right lead. The second contribution to $G^{d}$ describes double resonant tunneling processes, in which both quantum dots are involved (e.g., the electron tunnels first through $\mathrm{QD}_{1}$ and then through $\mathrm{QD}_{2}$ ). It is important to observe that in the presence of electron-electron interaction the exchange term also switches between the dots.

The on-site occupation number of the dots is given as usual by the relation:

$$
N_{i}=-\frac{i}{2 \pi} \int_{-2 t_{L}}^{2 t_{L}} d E \operatorname{Im} G_{i i}^{<}(E), \quad i=1,2 .
$$

We end this section by discussion a little bit more on the electron-electron interaction between the dots and the electrons in the wire (that is, between sites 1 and $\alpha$ and between sites 2 and $\beta$ ). By including this interaction one would have to add one more Hartree shift to each retarded self-energies $\Sigma_{I, 11}^{R, 1}$ and $\Sigma_{I, 22}^{R, 1}$. The two shifts contain the occupation numbers of the non-interacting contact sites (i.e their lesser Green functions $G_{\alpha \alpha}^{<}$and $G_{\beta \beta}^{<}$) which in the steady state are equal. Otherwise stated, the retarded self-energies acquire the same contribution from the corresponding contact site and the main difference is still given by the occupation numbers of the two dots which changes when we vary the gate potential on $\mathrm{QD}_{1}$ or the on-site energy $\varepsilon_{2}$. In what concerns the Coulomb interaction between the sites $\alpha$ and $\beta$ we can argue as well that it will not lead to qualitative changes in the results. Indeed, we shall have additional Hartree terms in the renormalized energies $\tilde{\varepsilon}_{\alpha, \beta}$ but these contributions are smaller than the one coming from the leads' self-energy - recall that we consider a strong coupling to the leads and then $t_{L}<U$.

\section{Results and discussion}

The parameter space for the system we consider here is considerably richer than in the case of a single-dot interferometer. The two couplings of the lateral dots control independently the widths of the two resonances $\tilde{\varepsilon}_{1}$ and $\tilde{\varepsilon}_{2}$. Moreover, their location can be tuned by varying the onsite energies $\varepsilon_{1}$ and $\varepsilon_{2}$ simulating thus the application of plunger gate potentials to each dot. These resonances add a significant contribution to the current only if $\operatorname{Re} \tilde{\varepsilon}_{i} \sim E$, for some energy values within the bias window. We consider here a symmetric bias with respect to the equilibrium chemical potential of the leads $\mu_{0}$ and we set $\mu_{0}=0$, that is $\mu_{L, R}= \pm V / 2$. In the numerical simulations the bias, the energy, the hopping constants on the leads, the coupling and interaction strengths will be expressed in terms of the hopping energy $t_{L}$ which is chosen as the energy unit. The current is given in $e t_{L} / h$ units.

As for the on-site energies of the upper sites we take $\varepsilon_{\alpha}=\varepsilon_{\beta}=-1.5$.

Before discussing the numerical results let us discuss the interference processes that lead to the Fano effect in the double $T$-shaped interferometer. The electronic trajectories within the interferometer fall within one of the following classes: (i) simple forward scattering in which electrons pass freely from one lead to another - this contribution is given by the term $G_{\alpha \beta}^{b}$ in equation (22); (ii) resonant tunneling through one dot only $\left(\mathrm{QD}_{1}\right.$ or $\left.\mathrm{QD}_{2}\right)$ and (iii) multiple tunneling processes implying both dots. If only one resonance (say $\tilde{\varepsilon}_{1}$ ) contributes to the transport, the interference is given essentially by $\left|G_{\alpha \beta}^{b}+G_{\alpha \alpha}^{b} t_{\alpha 1} G_{11}^{d} t_{1 \alpha} G_{\alpha \beta}^{b}\right|^{2}$ since the other terms from equation (22) are small. One recovers therefore the usual Fano line shape as a function of $\varepsilon_{1}$ (see the discussion of Fig. 2 below). In the following we shall investigate how this Fano line changes when the second quantum dot brings its own contribution to the transport. We find in particular that in the presence of the interdot Coulomb interaction the renormalized level of the second dot enters the bias window and provides a new transport channel.

In order to compare later on the effects of the first and second order contributions to the interaction self-energy we have performed first numerical simulations taking into 

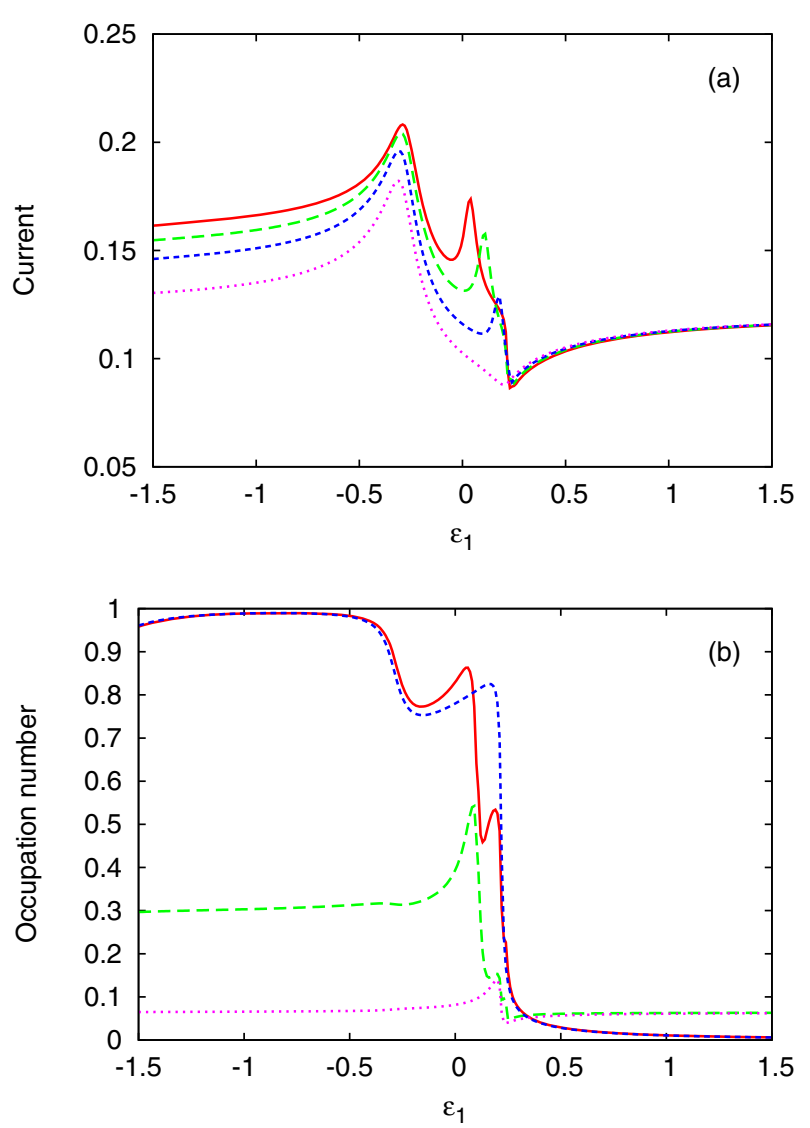

Fig. 2. (Color online) Fano line shapes of the current as a function of $\varepsilon_{1}$ for different values of $U$ at $\varepsilon_{2}=0.25$. $U=0.3-$ solid line, $U=0.2$ - long-dashed line, $U=0.1$ - dashed line, $U=0$ - dotted line. One notices the appearance of a second maxima on the right side of the Fano peak in the interacting case. (b) Occupation numbers on the dots $N_{1}$ and $N_{2}$ in interacting and noninteracting cases as a function of $\varepsilon_{1}$. Solid line - $N_{1}$ at $U=0.1$, dashed line $-N_{1}$ at $U=0$, long-dashed line - $N_{2}$ at $U=0.1$, dotted line $-N_{2}$ at $U=0$. The bias $V=0.5$ and $t_{1 \alpha}=t_{2 \beta}=0.25$.

account only $\Sigma_{I}^{1}$. The effect of $\Sigma_{I}^{2}$ will be discussed in relation to Figures 5 and 6 .

Figure 2a shows the current through the interferometer for different values of the interaction strength. We fix $\varepsilon_{2}=0.25$ and $t_{1 \alpha}=t_{2 \beta}=0.25$. When $U=0$ the usual Fano resonance is observed (the dotted line). The peak corresponds to constructive interference while the dip is associated with destructive interference between the background signal and the resonant contribution.

When the interaction strength is $U=0.1$ (dashed line) a second peak appears in the middle of the Fano line. The location of this peak changes with the interaction strength and the position of the Fano dip does not vary significantly. Also, on the left side of the peak the background signal is shifted to higher values. At first sight this additional peak is unexpected because it cannot be associated with the resonance in $\mathrm{QD}_{1}$. Another point which seems rather surprising is that on the right side of the Fano dip the interacting and noninteracting currents coincide while on the left side they do not.

In order to explain these results we give in Figure $2 b$ the occupation numbers $N_{1}$ and $N_{2}$ of the two dots as a function of $\varepsilon_{1}$ for $U=0$ and $U=0.1$. The occupation number of the first dot $N_{1}$ stays close to unity as long as $\varepsilon_{1}$ is far from the bias window (which covers the range $[-0.25: 0.25])$ and then decreases to zero. This behavior is typical for resonant tunneling: as the resonance enters the bias window the quantum dot participates in transport and finally empties when the resonance is pushed above the bias window. It is also clear that in the noninteracting case the second dot is almost empty $\left(N_{2}\right.$ is less than 0.1 ) and cannot bring an important contribution to the interference pattern.

The behavior of $N_{2}$ in the interacting case reveals more interesting features. Let us consider first the region $\varepsilon_{1}<-0.5$ when $\mathrm{QD}_{1}$ is off-resonance and almost filled. Since the leading order in $\Sigma_{22}^{R}$ is proportional to $N_{1}$ the level of the second dot acquires a shift due to the interdot interaction. The important point here is that if the distance between the 'bare' level $\varepsilon_{2}$ and the bias window is comparable to $\Sigma_{22}^{R}$ the 'renormalized' level is brought close to the bias window and therefore the transport regime of $\mathrm{QD}_{2}$ changes. One gets a supplementary contribution to the current which manifests itself as the up-shift of the background signal on the left side of the Fano resonances shown in Figure 2a. Also, the second dot accommodates more charge $N_{2} \sim 0.3$.

The physics becomes more complicated as the first dot is tuned to resonance (i.e when $\varepsilon_{1}$ is located within the bias window). On one hand the electrons escape into the the right lead after tunneling through the first dot. On the other hand, after tunneling out from $\mathrm{QD}_{1}$ a second resonant tunneling through $\mathrm{QD}_{2}$ is possible. This picture is confirmed by Figure $2 \mathrm{~b}$ which shows that in the interacting case $N_{2}$ increases and then decreases in Figure $2 \mathrm{~b}$. Finally, when the first dot empties the parameters of the second dot become the noninteracting ones because the contribution of the interaction self-energy $\Sigma_{I, 22}$ vanishes since it is proportional to $N_{1}$.

The above discussion suggests that the additional peak in Figure 2a is a consequence of a second interference process which involves both dots. The Fano line still develops when the level in $\mathrm{QD}_{1}$ enters the bias window and opens therefore a resonant path which interferes with the background component. However, as long as $N_{1}$ is large enough the resonance $\tilde{\varepsilon}_{2}$ is still located inside the bias window and therefore provides another resonant component of the transport to interfere with.

Indeed, note that the location of the second peak in Figure 2 a coincides with the peak in the occupation number of the second dot in Figure 2b. We have checked that as the interaction increases the second resonance enters further into the bias window, which explains why the distance between the two peaks decreases in Figure 2a. In the noninteracting case there is no second maximum in the current because the only visible interference at $\varepsilon_{1}=0.25$ 

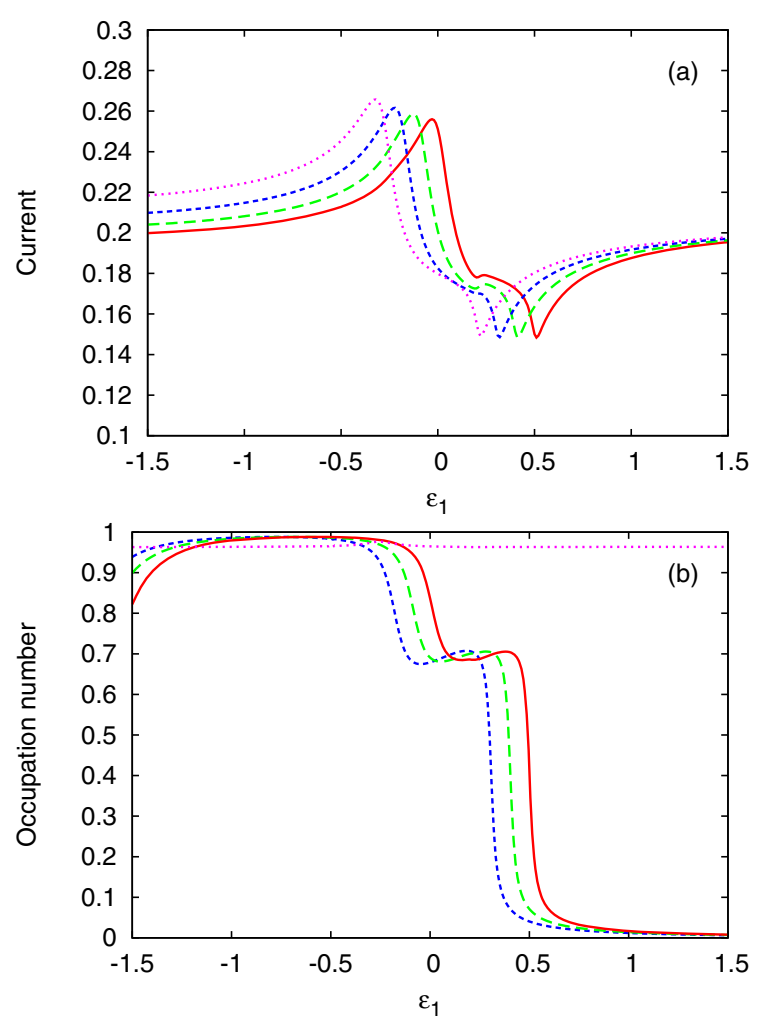

Fig. 3. (Color online) (a) The current through the interferometer as a function of $\varepsilon_{1}$ for different values of $U . U=0.3$ - solid line, $U=0.2$ - long-dashed line, $U=0.1$ - dashed line, $U=0$ - dotted line. (b) Occupation number on the dots $N_{1}$ and $N_{2}$ as a function of $\varepsilon_{1}$ at $\varepsilon_{2}=-0.5$. Solid line $-N_{1}$ at $U=0.3$, long-dashed line $N_{1}$ at $U=0.2$, dashed line $-N_{1}$ at $U=0.1$, dotted line $-N_{2}$ at $U=0.1$ (similar curves are obtained for other values of $U$ ). The other parameters are as in Figure 2.

involves the background signal and the resonant level in $\mathrm{QD}_{1}$.

The same values of the interaction strength are used to draw the curves in Figures 3a and 3b, but the level of the second dot is now set to $\varepsilon_{2}=-0.5$ which is well below the bias window. The current shows a totally different behavior, the interaction effect being mainly a shift of the Fano line and a reduction of the background signal on the left side of the resonance. The occupation numbers are presented in Figure 3b. Clearly in this case the second dot is completely occupied and does participate in transport because the noninteracting level $\varepsilon_{2}$ is too 'deep' and the renormalization induced by the interaction is not sufficient to make it active.

The numerical results presented above were obtained by taking into account the first order contribution to the interacting self-energy which describes only the elastic scattering processes. A natural question is what happens when higher order terms are included in the calculation. In the following we discuss the effect of the second order term. It is known that this term contains the bubble diagram that describes inelastic scattering involving electronhole pairs. This fact is also seen at the formal level in the 2 nd order contribution in the lesser self-energy which
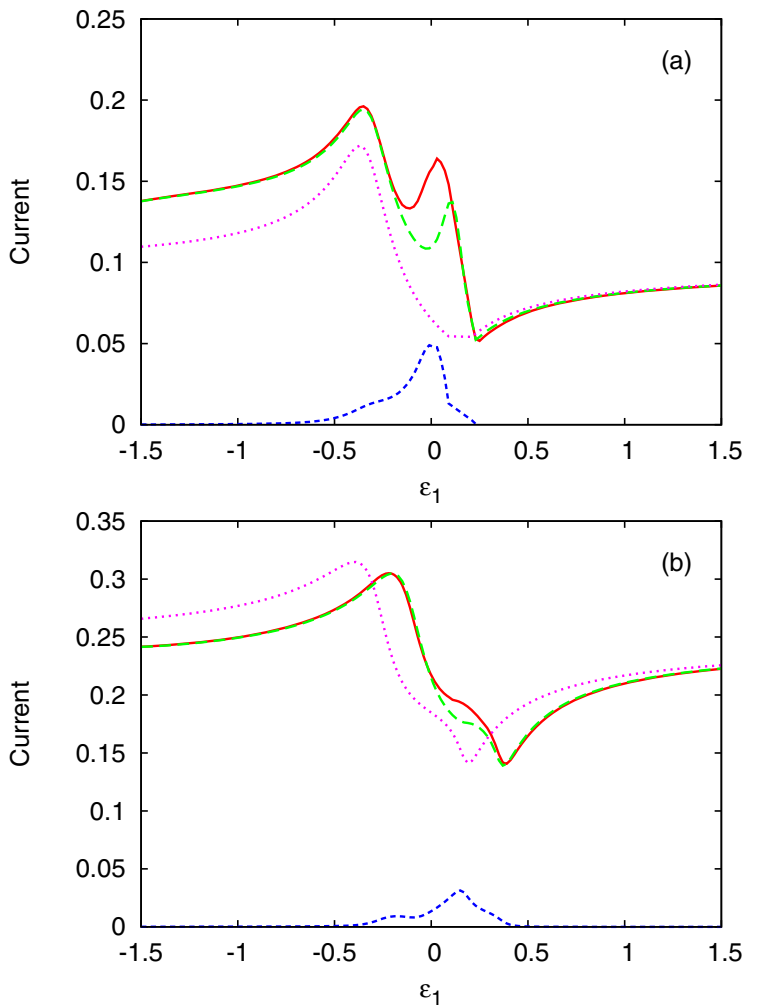

Fig. 4. (Color online) The effect of the second order contribution to the interaction self-energy. We show also the individual contribution of the second term in equation (5). Solid line - the total current, long-dashed line - the Landauer-like contribution, dashed line - the correction term, dotted line noninteracting total current. (a) $\varepsilon_{2}=0.25$, (b) $\varepsilon_{2}=-0.5$. The interaction strength here is $U=0.2$. The bias $V=0.5$ and $t_{1 \alpha}=t_{2 \beta}=0.25$.

depends on energy. Theoretical calculations show $[19,21]$ that the suppression of the Aharonov-Bohm oscillations and of the Fano line shape observed [22] in single-dot rings Coulomb-coupled to charge detectors can be qualitatively reproduced including just this second order contribution in the calculation. In the experimental setup of Buks et al. [22] the dephasing was observed only when the charge detector is subjected to a finite bias. Otherwise stated, as long as there is no charge transport in the detector the decoherence cannot appear. In order to understand the role of the intradot interaction on the Fano interference in a single dot or Coulomb coupled $T$-shaped interferometers we have recently adapted the random-phase approximation for the Keldysh formalism. [23]

The double $T$-shaped geometry considered here differs from the ones considered in reference [23]. There is no well defined detector, as both dots belong to the same system and they are allowed to exchange particles; also, the dots are driven out of equilibrium by the same bias. On the other hand, the $\Pi$ structure we are investigating here offers complex electronic trajectories. We find some interesting aspects related to the role of inelastic scattering in transport for this system as well. In Figure 4 we show the current obtained by using the first and second order 
(a)

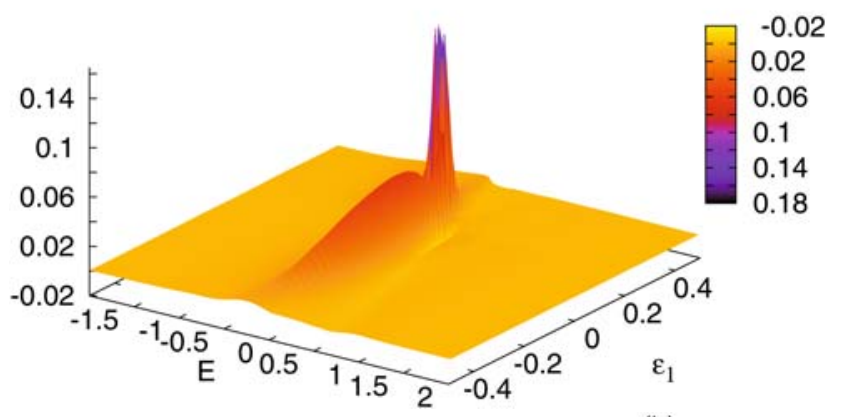

(b)

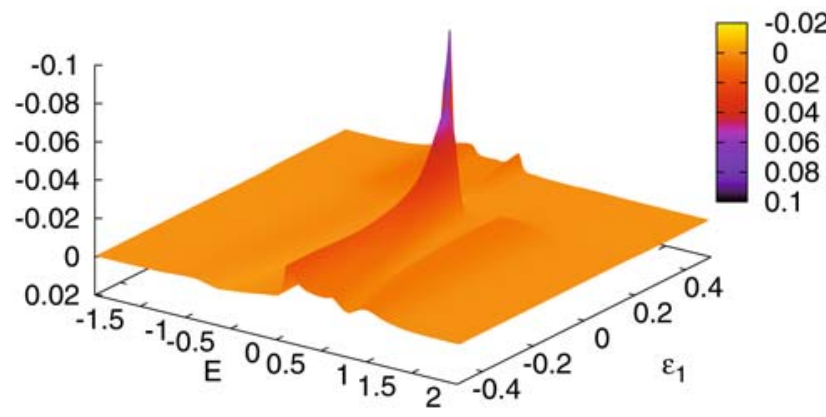

Fig. 5. (Color online) The imaginary part of the retarded interaction self-energy $-\Sigma_{I, 11}^{R}-(\mathrm{a})$ and $-\Sigma_{I, 22}^{R}-(\mathrm{b})$ computed up to the second order in $U$, as a function of $E$ and $\varepsilon_{1}$. We use the same parameters as in Figure $4 \mathrm{a}$.

terms in the interaction self-energy. We first take $\varepsilon_{4}=0.25$ (Fig. 4a), a value at which the second dot provides a second background contribution. With the inclusion of the second order self-energy the additional peak is higher and broader than the one in Figure 2a. This is mainly due to the correction term in equation (5) which adds to the Landauer-like current. It is important to observe that the contribution of this term significantly increases in the region of the additional peak, where both dots are active. As a consequence, the interference with the second background is the only one affected, the leftmost Fano peak being not modified. Figure $4 \mathrm{~b}$ presents the currents for $\varepsilon_{4}=-0.5$. In this case the second dot is almost filled (i.e., $N_{4} \sim 1$ ), does not contribute to the transport and the correction term is smaller. The two current curves do not differ much and it is clear that the effect of the second order interaction self-energy could be disregarded.

To complete our analysis we have also investigated the imaginary part of the second order retarded self-energy as a function of energies $E$ and $\varepsilon_{1}$ at $\varepsilon_{2}=0.25$. Figure 5 shows that when both $E$ and $\varepsilon_{1}$ lie in the bias window [-0.5:0.5] the imaginary parts of $\Sigma_{11}^{R}$ and $\Sigma_{22}^{R}$ exhibit two peaks. In Figure $5 \mathrm{a}$ one peak is rather small and broad while the second one is very sharp and can be clearly associated with the peak of the correction term in equation (5). The self-energy $\Sigma_{11}^{R}$ has only a single pronounced peak. Note that we actually show plots for the imaginary parts of $-\Sigma_{11}^{R}$ and of $\Sigma_{22}^{R}$ for clarity. We have checked that $\operatorname{Im} \Sigma_{12}^{R}$ gives a negligible contribution.
All these numerical data demonstrate that the inelastic scattering processes are important only when both dots contribute to the transport and only when there is an interference between the second background given by the right dot and the electronic trajectories associated with resonant tunneling through the left dot. Moreover, the main feature of the two side-coupled dots system (namely the existence of a second background contribution at suitable parameters for one of the dots) is not altered by the inelastic processes.

We end up with some comments on the particular features of the double $T$-shaped interferometer and on the applicability to experiments. First, it is clear that this geometry can be used to get some insight into the quantum interference processes in connected and interacting systems. We recall here that in the case of a mesoscopic interferometer Coulomb-coupled to a charge detector as in reference [22] there is no tunneling between the two subsystems, while in the double $T$-shaped interferometer the two quantum dots are also coupled via the leads. This fact leads to complex interference patterns as we have shown above. Secondly, the double $T$-shaped structure is quite different from the usual Aharonov-Bohm interferometer with one embedded dot in each arm. In the latter case the Fano interference appears only when both dots transmits. In the geometry considered here one can select a Fano line associated to one of the dots and control it by adjusting some parameters of the nearby dot.

\section{Conclusions}

We have theoretically investigated two transport regimes of a one-dimensional lead with two laterally coupled single-level quantum dots (a double $T$-shaped interferometer), in the presence of interdot Coulomb interaction. The first regime involves both dots in the transport: one (reference) dot has an energy level which is sufficiently close to the bias window of the leads while the second one can be set on resonance by varying a plunger gate voltage. This situation could be met if the charge sensing effect of the nearby dot leads to an upper shift of the levels in the reference dot. Alternatively, the gate potential applied on the reference dot should be set to a suitable value. We emphasize both at the formal level and by numerical simulations that in this regime a second interference appears due to a supplementary background contribution of the reference dot. Consequently, an additional peak develops in the Fano line shape of the current. In the second regime only the reference dot is off-resonance and its effect in transport reduces to a Hartree shift of the Fano line.

A double $T$-shaped system like the one considered here can easily be realized in future experiments [24] and should provide important insights on the control of quantum interference and on the decoherence induced by the mutual Coulomb interaction between the dots. The two regimes and the crossover between them can be experimentally observed by tuning the gate potentials of the lead-coupling strength. We would like to point out the 
paper by Malyshev et al. [25] who study the propagation of a Gaussian pulse through a similar configuration, in the absence of electron-electron interaction. We have also discussed the effect of the inelastic scattering processes by computing the interaction self-energy up to the second order in the Coulomb coupling strength.

B.T. is supported by TUBITAK (No. 106T052) and TUBA. V.M. and I.V.D. acknowledge financial support by TUBITAK-BIDEB and CEEX Grant D11-45/2005.

\section{References}

1. S. Katsumoto, J. Phys.: Condens. Matter 19, 233201 (2007)

2. T. Ihn, M. Sigrist, K. Ensslin, W. Wegscheider, M. Reinwald, New J. Phys. 9, 111 (2007)

3. K. Kobayashi, H. Aikawa, A. Sano, S. Katsumoto, Y. Iye, Phys. Rev. B 70, 035319 (2004)

4. U. Fano, Phys. Rev. 124, 1866 (1961)

5. K. Kobayashi, H. Aikawa, S. Katsumoto, Y. Iye, Phys. Rev. Lett. 88, 256806 (2002)

6. M. Sato, H. Aikawa, K. Kobayashi, S. Katsumoto, Y. Iye, Phys. Rev. Lett. 95, 066801 (2005)

7. T.-S. Kim, S. Hershfield, Phys. Rev. B 63, 245326 (2001)

8. R. Franco, M.S. Figueira, E.V. Anda, Phys. Rev. B 67, 155301 (2003); R. Franco, M.S. Figueira, E.V. Anda, Phys. Rev. B 73, 195305 (2006)

9. P.S. Cornaglia, D.R. Grempel, Phys. Rev. B 71, 075305 (2005)
10. B.H. Wu, J.C. Cao, K.-H. Ahn, Phys. Rev. B 72, 165313 (2005)

11. O. Entin-Wohlman, A. Aharony, Y. Meir, Phys. Rev. B 71, 035333 (2005)

12. R. Zitko, J. Bonca, J. Phys.: Condens. Matter 19, 255205 (2007)

13. D. Sztenkiel, R. Swirkowicz, J. Phys.: Condens. Matter 19, 176202 (2007); D. Sztenkiel, R. Swirkowicz, J. Phys.: Condens. Matter 19, 386224 (2007)

14. D.N. Son, N. Arboleda, Jr, W.A. Dino, H. Kasai, Eur. Phys. J. B 57, 27 (2007)

15. J. Ma, B. Dong, X.L. Lei, Eur. Phys. J. B 36, 599 (2003)

16. Y.-S. Liu, H. Chen, X.-F. Yang, J. Phys.: Condens. Matter 19, 246201, (2007)

17. C. Caroli, R. Combescot, P. Nozieres, D. Saint-James, J. Phys. C 4, 916 (1971)

18. H. Haug, A.-P. Jauho, Quantum Kinetics in Transport and Optics of Semiconductors (Springer, Berlin, 1996)

19. V. Moldoveanu, M. Ţolea, B. Tanatar, Phys. Rev. B 75, 045309 (2007)

20. V. Moldoveanu, M. Tolea, B. Tanatar, Physica E 40, 1102 (2008)

21. A. Silva, S. Levit, Phys. Rev. B 63, 201309 (2001)

22. E. Buks, R. Schuster, M. Heiblum, D. Mahalu, V. Umansky, Nature (London) 391, 871 (1998)

23. V. Moldoveanu, B. Tanatar, Phys. Rev. B 77, 195302 (2008)

24. Actually a very similar structure was investigated by Sasaki et al., Phys. Rev. B 73, 161303(R) (2006) in the context of the Kondo effect

25. A.V. Malyshev, P.A. Orellana, F. Domínguez-Adame, Phys. Rev. B 74, 033308 (2006) 